\title{
Using a Genetic Algorithm to Solve a Bi-Objective WWTP Process Optimization
}

\author{
Lino Costa, Isabel A. C. P. Espírito-Santo, Edite M. G. P. Fernandes, and Roman \\ Denysiuk
}

\begin{abstract}
When modeling an activated sludge system of a wastewater treatment plant (WWTP), several conflicting objectives may arise. The proposed formulation is a highly constrained bi-objective problem where the minimization of the investment and operation costs and the maximization of the quality of the effluent are simultaneously optimized. These two conflicting objectives give rise to a set of Pareto optimal solutions, reflecting different compromises between the objectives. Population based algorithms are particularly suitable to tackle multi-objective problems since they can, in principle, find multiple widely different approximations to the Pareto-optimal solutions in a single run. In this work, the formulated problem is solved through an elitist multi-objective genetic algorithm coupled with a constrained tournament technique. Several trade-offs between objectives are obtained through the optimization process. The direct visualization of the trade-offs through a Pareto curve assists the decision maker in the selection of crucial design and operation variables. The experimental results are promising, with physical meaning and highlight the advantages of using a multi-objective approach.
\end{abstract}

\section{Multi-objective Optimization}

We apply the Multi-objective Elitist Genetic Algorithm (MEGA), described in [3] to the WWTP multi-objective optimization problem. This approach, in contrast to other algorithms, does not require any differentiability or convexity conditions of the search space. Moreover, since it works with a population of points, it can find,

Lino Costa - Isabel A. C. P. Espírito-Santo - Edite M. G. P. Fernandes

Department of Production and Systems, University of Minho, Campus de Gualtar, 4710-057 Braga, Portugal e-mail: \{lac,iapinho,emgpf $\}$ dps.uminho.pt

Roman Denysiuk

Algoritmi R\&D Center, University of Minho, Campus de Gualtar, 4710-057 Braga, Portugal email: denysiukr@gmail.com 
in a single run, multiple approximations to the solutions of the Pareto optimal set without the need of fixing any weights and a well distributed representation of the Pareto optimal frontier induced by the use of diversity-preservation mechanisms. We now shortly describe some technical features and the parameters of this algorithm 1 .

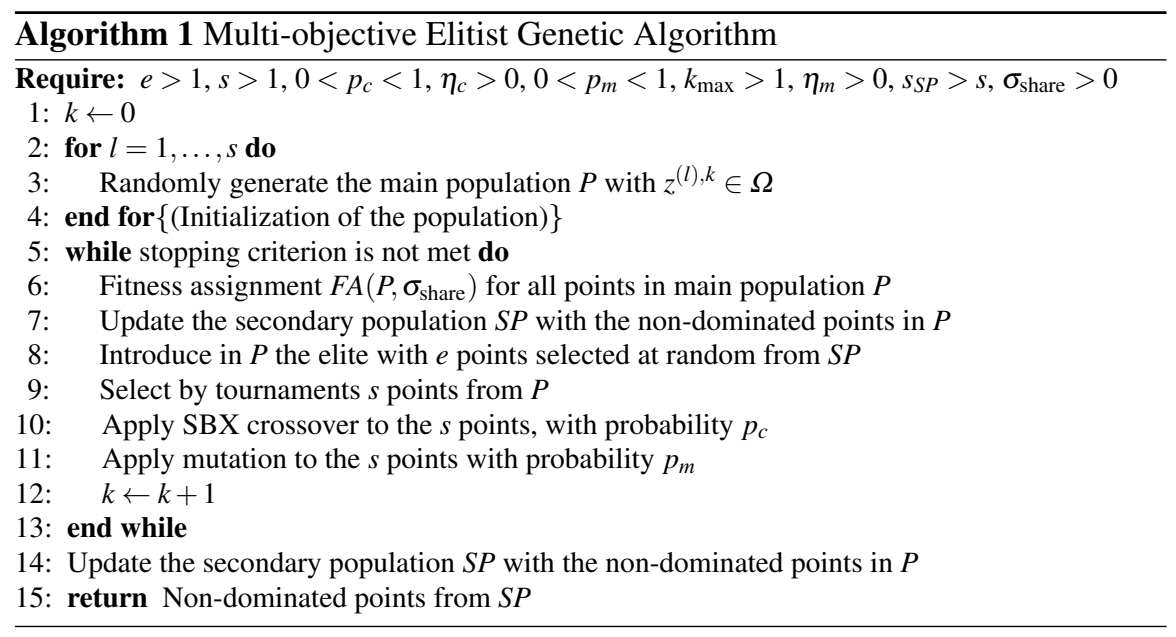

MEGA starts from a population of points $P$ of size $s$. In our implementation, a real representation is used since we are leading with a continuous problem. Additionally, a secondary population $S P$ that archives potential Pareto optimal solutions found so far during the search process is maintained. The elitist technique implemented is based on the secondary population with a fixed parameter $e(e \geq 1)$ that controls the elitism level, i.e., $e$ is the maximum number of non-dominated solutions of the secondary population that will be introduced in the main population. These non-dominated solutions will effectively participate in the search process that is performed using the points of the main population.

In order to handle constraints, we implemented the constrained tournament method in which a new dominance relation is defined [4]. A solution $x \in \mathbb{R}^{n}$ constrain-dominates $y \in \mathbb{R}^{n}$, i.e., $x \prec_{c} y$ if and only if: $x$ is feasible and $y$ is not; $x$ and $y$ are unfeasible, but $x$ has a smaller constraint violation; $x$ and $y$ are feasible, $x$ dominates $y$, i.e., $x \prec y$.

Solutions are evaluated according to a fitness assignment function $F A\left(P, \sigma_{\text {share }}\right)$ that is based on the constraint-dominance relation between points. All solutions are ranked in terms of dominance defining several fronts. Therefore, all non-dominated solutions in the main population $P$ will constitute the first front to which is assigned a rank equal to 1 . Successively, the same procedure is applied to the remaining points defining several fronts with increasing ranks. In order to maintain diversity, a sharing scheme depending on an initial parameter $\sigma_{\text {share }}$ is applied to the solutions belonging 
to the same front. For this purpose, an adaptive sharing scheme on objective space was adopted for diversity preservation as described in [2].

Non-dominated points in main are archived in $S P$. The $S P$ update implies the determination of Pareto optimality of all solutions stored so far, in order to eliminate those that became dominated. As the size of $S P$ grows, the time to complete this operation may become significant. So, in order to prevent the growing computation times, in general, a maximum $s_{S P}>s$ size is imposed.

A tournament selection that guarantees that better points are more likely to be selected was used to select points from the main population. New points in the search space are generated by the application, with probability $p_{c}$, of a Simulated Binary Crossover (SBX) [3,2] that simulates the working principle of single-point crossover operator for binary strings. A Polynomial Mutation is applied, with a probability $p_{m}$, to the points produced by the crossover operator. Mutation introduces diversity in the population since crossover, exclusively, could not assure the exploration of new regions of the search space.

The search ends when a given stopping criterion is satisfied. The best approximations to the Pareto-optimal set are archived in $S P$.

\section{The case study: WWTP optimal design}

The system under study consists of an aeration tank, where the biological reactions take place, and a secondary settler for the sedimentation of the sludge and clarification of the effluent. To describe the aeration tank we chose the activated sludge model n.1, described by Henze et al. [8]. The tank is considered to operate in steady state and as a completely stirred tank reactor and the generic equation for a mass balance in these conditions is

$$
\frac{Q}{V_{\mathrm{a}}}\left(\xi_{\text {in }}-\xi\right)+r_{\xi}=0,
$$

where $Q$ is the flow that enters the tank, $V_{\mathrm{a}}$ is the aeration tank volume, $\xi$ and $\xi_{\text {in }}$ are the concentrations of the components, particulates or solubles, around witch the mass balances are being made inside the reactor and on entry, respectively. $r_{\xi}$ is obtained by the Peterson Matrix [8] and is the process reaction rate.

Another set of constraints is concerned with the secondary settler. When the wastewater leaves the aeration tank, the treated water should be separated from the biological sludge, otherwise, the chemical oxygen demand would be higher than it is at the entry of the system. The ATV design procedure [5] contemplates the peak wet weather flow events, during which there is a reduction in the sludge concentration and is based on very simple empirical relations.

Besides the ATV procedure, the double exponential model [9] is also used to describe the sedimentation process [7]. This model assumes a one dimensional settler, in which the tank is divided into 10 layers of equal thickness. It assumes that no biological reactions take place, meaning that the dissolved matter concentration is 
maintained across all the layers. Only vertical flux is considered and the solids are uniformly distributed across the entire cross-sectional area of the feed layer. This model is based on a traditional solids flux analysis but the flux in a particular layer is limited by what can be handled by the adjacent layer. The settling function is given by

$$
v_{s, j}=\max \left(0, \min \left(v_{0}^{\prime}, v_{0}\left(e^{-r_{h}\left(T S S_{j}-f_{n s} T S S_{a}\right)}-e^{-r_{p}\left(T S S_{j}-f_{n s} T S S_{a}\right)}\right)\right)\right)
$$

where $v_{s, j}$ is the settling velocity in layer $j, T S S_{j}$ is the total suspended solids concentration in each of the ten considered layers and $v_{0}, v_{0}^{\prime}, r_{h}, r_{p}$ and $f_{n s}$ are settling parameters. This model introduces discontinuities in the problem.

The other important group of constraints are a set of linear equalities and defines composite variables. In a real system, some state variables are, most of the time, not available for evaluation. Thus, readily measured composite variables are used instead. This includes the chemical oxygen demand $(C O D)$, total suspend solids $(T S S)$ and total nitrogen $(N)$, to name the more important.

The system behavior, in terms of concentration and flows, may be predicted by balances. In order to achieve a consistent system, these balances must be done around the entire system and not only around each unitary process. They were done to the suspended matter, dissolved matter and flows. For example, to the soluble compounds, represented by $S$ ? we have

$$
(1+r) Q_{\mathrm{inf}} S_{\text {?ent }}=Q_{\mathrm{inf}} S_{\text {?inf }}+r Q_{\mathrm{inf}} S_{?}
$$

where $r$ is the recycle rate and $Q_{\text {? }}$ the volumetric flows. As to the subscripts, inf concerns the influent wastewater and ent the entry of the aeration tank.

It is also necessary to add some system variables definitions, in order to define the system correctly. All the variables are considered non-negative, although more restricted bounds are imposed to some of them due to operational consistencies. As an example, the amount of soluble oxygen in the aeration tank must be at least 2 g/ML. These conditions define a set of simple bounds on the variables.

Finally, the quality of the effluent has to be imposed. The quality constraints are usually derived from law restrictions. The most used are related with limits in the Chemical Oxygen Demand (COD), Nitrogen $(N)$ and Total Suspended Solids (TSS) at the effluent. In mathematical terms, these constraints are defined by portuguese laws as $C O D_{\text {ef }} \leq 125, N_{\text {ef }} \leq 15$ and $T S S_{\text {ef }} \leq 35$. We refer to [6] for more details.

The first objective function of the problem represents the total cost and includes both investment and operation costs. The operation cost is usually on annual basis, so it has to be updated to a present value using the adequate economic factors of conversion. Each term in the objective function is based on the basic model $C=a Z^{b}$ [10], where $a$ and $b$ are the parameters to be estimated, $C$ is the cost and $Z$ is the characteristic of the unitary process that most influences the cost. The parameters $a$ and $b$ are estimated by the least squares technique, using real data collected from a WWTP building company. Summing up the terms from all the costs in all considered units, we get the following Total Cost $(T C)$ objective function that depends on the 
aeration tank volume $\left(V_{\mathrm{a}}\right)$, the air flow $\left(G_{\mathrm{S}}\right)$, the sedimentation area $\left(A_{\mathrm{S}}\right)$ and depth (h).

$T C\left(V_{\mathrm{a}}, G_{\mathrm{S}}, A_{\mathrm{s}}, h\right)=174.2 V_{\mathrm{a}}^{1.07}+12487 G_{\mathrm{S}}^{0.62}+114.8 G_{\mathrm{S}}+955.5 A_{\mathrm{s}}^{0.97}+41.3\left(A_{\mathrm{s}} h\right)^{1.07}$.

The second objective function is the Quality Index $(Q I)$ defined by the BSM1 model [1] and gives a measure of the amount of daily pollution. It depends on the quality of the effluent in terms of TSS, COD, biochemical oxygen demand (BOD), total Kjeldahl nitrogen $(T K N)$, nitrate and nitrite nitrogen $\left(S_{\mathrm{NO}}\right)$ and the effluent flow $\left(Q_{\mathrm{ef}}\right)$. The obtained function is

$Q I\left(T S S, C O D, B O D, T K N, N O, Q_{\mathrm{ef}}\right)=\left(2 T S S+C O D+2 B O D+20 T K N+2 S_{\mathrm{NO}}\right) \frac{Q_{\mathrm{ef}}}{1000}$.

\section{Numerical results and Conclusions}

The mathematical model has 2 objective functions, 71 parameters, 113 variables, 103 equality constraints and one inequality constraint. All the variables are bounded below and above. The stoichiometric, kinetic and operational parameters are the default values presented in the GPS-X simulator [11], and they are usually found in real activated sludge based plants. The MatLab implementation of the problem is available from the webpage http://www.norg.uminho.pt/iapinho/proj.htm under "Bi-objective WWTP Project".

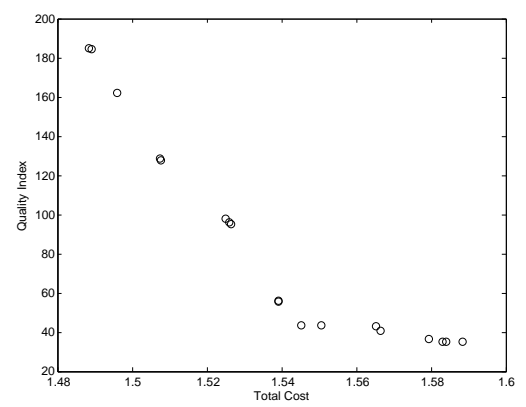

\begin{tabular}{c|cc} 
& $T C_{\min }$ & $Q I_{\min }$ \\
\hline$V_{\mathrm{a}}$ & 1567 & 1567 \\
$G_{\mathrm{S}}$ & 100 & 100 \\
$A_{\mathrm{s}}$ & 816 & 813 \\
$h$ & 3.3 & 4.9 \\
$T C$ & 1.49 & 1.59 \\
$Q I$ & 185 & 35
\end{tabular}

Fig. 1 Pareto curve for the Total Cost and Quality Index, and optimal values for the most important variables

The MEGA algorithm was coded in MatLab programming language and the numerical results were obtained with a Intel Core2 Duo CPU $1.8 \mathrm{GHz}$ with $2 \mathrm{~GB}$ of memory. The MEGA parameters are: $s=40, e=4, p_{c}=0.9, \eta_{c}=20, p_{m}=1 / 113$, $\eta_{m}=20, s_{S P}=\infty$ and $\sigma_{\text {share }}=0.1$. The maximum number of objective function evaluations is 50000. An initial value, $x^{0}$, provided by the GPS-X simulator [11] with the real influent data was introduced in the initial population. Several exper- 
iments were conducted without introducing this initial point in the population and the algorithm failed to achieve a feasible point within the maximum number of objective function evaluations.

Figure 3 shows the Pareto optimal front defined by the approximations to the Pareto optimal solutions. In this figure, the compromise solutions between $Q I$ and $T C$ are plotted. It is also presented the results for the most important decision variables of the limit solutions from the Pareto front $\left(T C_{\min }\right.$ and $\left.Q I_{\min }\right)$, namely, the aeration tank volume, the air flow, the area and depth of the secondary settler, as well as $C O D, T S S$ and $N$ at the effluent. The total computational time is about 190 seconds. We can observe that the non-dominated solutions obtained are viable and have physical meaning, highlighting the superiority of the bi-objective approach in terms of computational demands. The obtained WWTP designs represent compromises that are economically attractive with convenient quality indexes and satisfy the law limits. Moreover, these limits in terms of $C O D$ and $T S S$ are below the law limits, showing the robustness of the solution. Although the obtained WWTP designs are attractive, in the future we intend to propose a multi-objective approach with more than two objectives. For example, air flow requirements and chemicals addition will be considered.

\section{References}

1. J. Alex, L. Benedetti, J. Copp, K.V. Gernaey, U. Jeppsson, I. Nopens, M.N. Pons, C. Rosen, J.P. Steyer and P. Vanrolleghem (2008) Benchmark Simulation Model no. 1 (BSM1). Technical Report prepared by the IWA Taskgroup pn Benchmarking of Control Strategies for WWTPs.

2. L. Costa, and P. Oliveira (2003) An Adaptive Sharing Elitist Evolution Strategy for Multiobjective Optimization. Evolutionary Computation, 11(4), 417-438.

3. L. Costa and P. Oliveira (2003) An elitist genetic algorithm for multiobjective optimization, in M.G.C. Resende and J.P. de Sousa (eds.), Metaheuristics: Computer Decision-Making, pp. 217-236, Kluwer Academic Publishers.

4. K. Deb (2000) An efficient constraint handling method for genetic algorithms, Computer Methods in Applied Mechanics and Engineering, 186(2-4), 311-338.

5. G.A. Ekama, J.L. Barnard, F.H. Günthert, P. Krebs, J.A. McCorquodale, D.S. Parker and E.J. Wahlberg (1978) Secondary Settling Tanks: Theory, Modeling, Design and Operation. Technical Report 6, IAWQ - International Association on Water Quality.

6. I.A.C.P. Espírito Santo, E.M.G.P. Fernandes, M.M. Araújo and E.C. Ferreira (2006) How Wastewater Processes can be Optimized using LOQO. Lecture Notes in Economics and Mathematical Systems, A. Seeger (ed.), Springer-Verlag 563:435-455.

7. I.A.C.P. Espírito Santo, E.M.G.P. Fernandes, M.M. Araújo and E.C. Ferreira (2006) On the Secondary Settler Models Robustness by Simulation. WSEAS Transactions on Information Science and Applications 3:2323-2330.

8. M. Henze, C.P.L Grady Jr, G.V.R Marais and T. Matsuo (1986) Activated Sludge Model no. 1, Technical Report 1, IAWPRC Task Group on Mathematical Modelling for Design and Operation of Biological Wastewater Treatment.

9. I. Takács, G.G. Patry and D. Nolasco (1991) A Dynamic Model of the Clarification-Thickening Process, Water Research 25:1263-1271.

10. D. Tyteca, Y. Smeers and E.J. Nyns (1977) Mathematical Modeling and Economic Optimization of Wastewater Treatment Plants. CRC Critical Reviews in Environmental Control 8:1-89.

11. GPS-X Technical Reference (2002). Hydromantis, Inc. 